\title{
Aerodynamic characteristics of hoverflies during hovering flight
}

\author{
Fang-Bao Tian ${ }^{1 \mathrm{a}}$, John Young ${ }^{\mathrm{a}}$, Joseph C. S. Lai ${ }^{\mathrm{a}}$, Simon M. Walker ${ }^{\mathrm{b}}$, Graham K. Taylor ${ }^{\mathrm{b}}$, \\ Adrian L. R. Thomas ${ }^{b}$ \\ ${ }^{a}$ School of Engineering and Information Technology, University of New South Wales, Canberra, ACT 2600, \\ Australia \\ ${ }^{b}$ Department of Zoology, University of Oxford, Oxford OX1 3PS, UK
}

\begin{abstract}
Aerodynamic characteristics of hoverflies during hovering flight are studied with a threedimensional sharp-interface immersed boundary method. The wing of hoverfly-is modelled a rectangular generic wing. Simulations are conducted in two groups: One group of simulations are conducted to determine the equilibrium kinematics of the wings with and without alula; the other group of simulations are conducted to study the effects of pitching phase differences between the wing and the alula on the performance of the wings. The forces, aerodynamic power, efficiency and vortical structures are discussed in detail. It is found that the wing without alula experiences larger angle of attack at equilibrium-flight. This difference $5 \%$ more aerodynamic power compared to the wing with alula. By performing simulations of the wing without alula, and wings with alula flapping $45^{\circ}$ ahead, in phase with and $45^{\circ}$ behind of the wing, we find that the wing with alula flapping in phase produces largest lift, but its efficiency is lowest. Vortical structure analysis shows that the vortical structures of the wing with alula flapping $45^{\circ}$ ahead are similar to those of the wing without alula. The alula provides stabilizing effect on the leading edge vortex for the wings with alula flapping $45^{\circ}$ ahead in phase.
\end{abstract}

Key words: Sharp-interface immersed boundary method; Insect flight; Alula; Equilibrium flight.

\footnotetext{
${ }^{1}$ Correspondence author. Email: f.tian@adfa.edu.au and onetfbao@gmail.com
} 


\section{Introduction}

The mechanisms of insect flight have become a central issue for researchers and engineers wishing to develop micro air vehicles (MAVs) with capabilities exceeding existing fixed and rotary wing designs. Areas where aerial animals (such as bumblebees and hoverflies) out-perform conventional man-made vehicles include high efficiency, maneuverability, stability, gust resistance and load capacity, and low noise signature. The aerodynamics differ considerably between these animals and conventional fixed or rotary wing vehicles. These differences may be attributed to the effects of the complex locomotion, Reynolds number and biomaterial properties (e.g. the-flexibility and the-inertia), and unsteady aerodynamics. Therefore, great efforts have been made in the past few decades to study this subject (Shyy et al., 2008, 2010; Wu, 2011; Deng et al., 2013).

Experimental measurements and observations have been performed to measure and/or observe the biomaterial properties, morphology, kinematics, dynamics and neural signal (Hertel, 1966; Ellington, 1984b,c; Dickinson and Lighton, 1995; Wootton, 1999; Dickinson and Lighton, 2000; Wootton et al., 2003; Sane, 2003; Fry et al., 2003; Iosilevskii and Joel, 2013). A common strategy for an insect flying in the air is to use its wings to make stroke, pitching and deviation (Alexander, 1993; Vogel, 1994; Shyy et al., 2008). Based on observations and measurements of animal locomotion, simple models can be used in the ex-

periments to study the mechanisms (Ellington et al., 1996; Dickinson et al., 1999; Birch and Dickinson, 2002; Prempraneerach et al., 2003; Triantafyllou et al., 2004). Theoretical analyses have also been proposed to study biofluid dynamics of the animal flight. For example, Lighthill (Lighthill, 1973) and Edwards and Cheng (Edwards and Cheng, 1981) presented the two-dimensional inviscid analysis of the Weis-Fogh mechanism (Weis-Fogh, 1973) of insect flight; Ellington (Ellington, 1984a,c) proposed a scheme to incorporate wing rotation with translation in a quasi-steady model. The complex geometries, motions and flow fields make both experimental measurements and theoretical analyses challenging. Numerical modelling is an alternative to investigate this topic.

Hovering flight is the most demanding type of sustained locomotion, as it is the most energetically expensive form of flight and it is very hard to remain stationary (Walker et al., 
2010). The horfly is noted for its excellent hovering flight skills. A notable morphological feature of alula which is hinged at the base of the wings and accounts for up to 10 per cent of the total wing area (Walker et al., 2012). Though the flapping kinematics and aerodynamics of hoverflies have been studied by several researchers (see for example Refs. (Walker et al., 2010; Mou et al., 2011; Mou and Sun, 2012; Walker et al., 2012)), the effects of kinematies and alula are still not well understood. By using high-speed camera to record free-flying hoverflies and to analyze the relationship between the alula flipping and wing kinematics, Walker et al. (2012) hypothesized that the observed changes in the aerodynamic forces are due to associated changes in wing kinematics (especially the change in stroke angle) rather than the aerodynamic effects of the alula. However, it is difficult to ascertain the aerodynamic contributions of the alula and all parameters that are changed associated with alula flipping.

In this work, a generic wing model is used to investigate the effects of the alula on the equilibrium kinematics and the flight performance by using the sharp-interface immersed boundary method Tian et al. (2014a). The equilibrium kinematics, forces, power requirement and vortical structures are discussed in detail.

The rest of this paper is organized as follows. Section 2 describes the physical problem, mathematical formulation and numerical method. The numerical results and discussion are presented in Section 3. Finally, concluding remarks are provided in Section 4.

\section{Physical problem and numerical method}

\subsection{Flapping motion of a wing}

The physical problem considered here is shown in Fig. 1 where a generic wing strokes in the stroke plane about the pivot point and pitches about the pitching axis. The

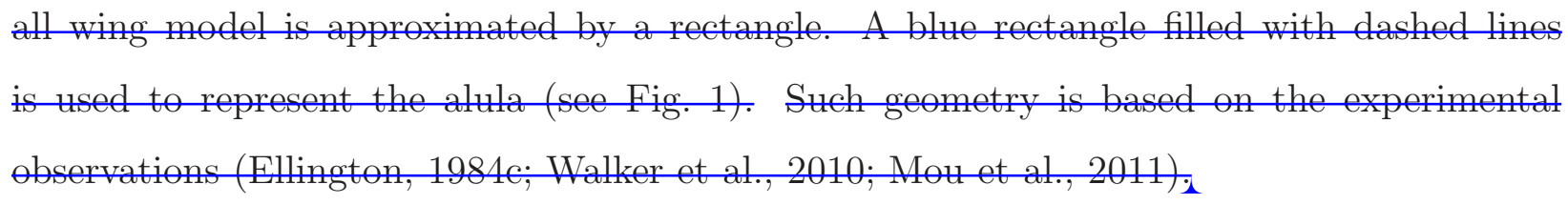




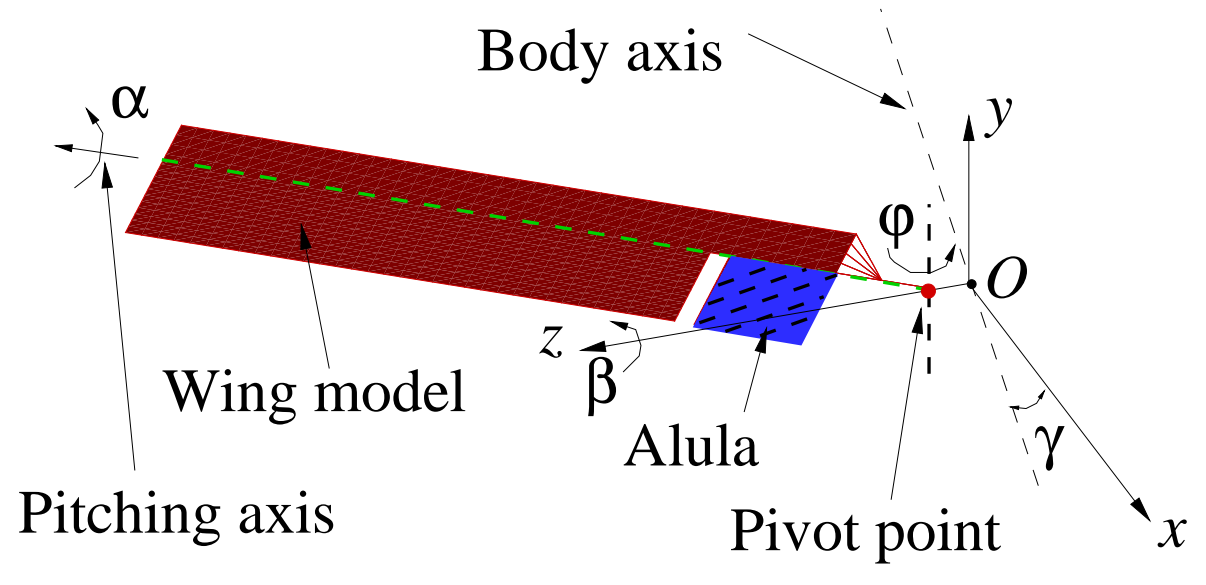

Figure 1: Definitions of the angles of the flapping wing. $(x, y, z)$ are coordinates in a system with its origin at the center between two wing pivot points, $z$-axis pointing to the side of the insect and $x$ - $z$ plane coinciding with the horizontal plane. The blue rectangle filled with dashed lines is the alula.

The Wing motion is an important part of the simulations. Based on observations (Walker et al., 2010; Mou et al., 2011), the flapping motion of a hoverfly wing during hovering mainly consists of two parts: the strolening, which are denoted by $\varphi$ and $\alpha$ (as shown in Fig. 1). The stroke motion is approximated by a simple harmonic function

$$
\varphi=\varphi_{0}+0.5 \Phi \sin (2 \pi f t)
$$

where $f$ is the frequency, $\varphi_{0}$ is the mean stroke angle, and $\Phi$ is the maximum stroke angle. The pitching angle takes a constant value at the mid position of the downstroke or upstroke. The constant value is represented by $\alpha_{d}$ for the downstroke and $\alpha_{u}$ for the upstroke. Around stroke reversal, the wing rotates and $\alpha$ changes with time. $\alpha$ during the supination at $m$-th cycle is described by

$$
\alpha=\alpha_{d}+A\left\{\left(t-t_{1}\right)-\frac{\Delta t_{r}}{2 \pi} \sin \left[2 \pi\left(t-t_{1}\right) / \Delta t_{r}\right]\right\}, t_{1} \leq t \leq t_{1}+\Delta t_{r}
$$

where $\Delta t_{r}$ is the time duration of wing pitching during the stroke reversal, $t_{1}$ is the time 
when the wing-pitching starts, and $A$ is a constant determined by

$$
A=\left(180^{0}-\alpha_{u}-\alpha_{d}\right) / \Delta t_{r}, t_{1}=(m-1) T+\zeta T,
$$

where $T$ is the flapping period, and $\zeta$ is a number between 0 and $1 . \alpha$ during the pronation at $m$-th cycle can be obtained by a similar method. Such descriptions are the same as those used in Refs. (Xiong et al., 2008; Mou and Sun, 2012). From Fig. 1 and Eqs. (1)-(3), it is noted that parameters $\Phi, f, \zeta, \Delta t_{r}, \alpha_{d}, \alpha_{u}, \varphi_{0}$, the stroke plane angle $\beta$, and body angle $\gamma$ are required to describe the wing kinematics. The kinematics of alula can be described by Eqs (1) and (2) with a delay in phase. Three phase delays are considered: in-phase, $45^{\circ}$ ahead and behind the wing.

\subsection{Mathematical formulation}

For most insects, the velocities of wing tips are much smaller than the acoustic velocity of the fluid (Deng et al., 2013) and the fluid motion can be described by the incompressible Navier-Stokes equations,

$$
\begin{aligned}
& \frac{\partial v_{i}}{\partial x_{i}}=0, \\
& \frac{\partial v_{i}}{\partial t}+\frac{\partial v_{j} v_{i}}{\partial x_{j}}=-\frac{1}{\rho} \frac{\partial p}{\partial x_{i}}+\nu \frac{\partial^{2} v_{i}}{\partial x_{j}^{2}},
\end{aligned}
$$

where $v_{i}$ is the velocity, $\rho$ is the density, $\nu$ is the kinematic viscosity, and $p$ is the pressure. In this work, the structure kinematics is prescribed, i.e.

$$
\mathbf{v}=\mathbf{v}_{b} \quad \text { at } \quad \mathbf{X}=\mathbf{X}_{b}
$$

where $\mathbf{X}_{b}$ and $\mathbf{v}_{b}$ are respectively the prescribed position and velocity of the boundary.

In this work, a sharp-interface immersed boundary method is used to solve the above equations. Details of this method and its validation can be found in Appendix A.

\section{Results and discussion}

In order to investigate the effects of the alula on the flight performance, a generic wing model is employed in the simulations, as shown in Fig. 1. The wing chord length is $c=$ 
$1.71 \mathrm{~mm}$, the wing length is $L=6.93 \mathrm{~mm}$, the pitching axis is located at quarter chord from the leading ledge, the alula length along wing span is $L_{a}=1.09 \mathrm{~mm}$, the gap between the wing and alula is $g_{a}=0.2 \mathrm{~mm}$, the pivot point is located at $0.94 \mathrm{~mm}$ from the wing root. These geometric parameters are ample 2011).

The parameters of dry air at a pressure of one atmosphere and $15^{\circ} \mathrm{C}$ are used for the fluid. Specifically, $\rho=1.225 \mathrm{~g} / \mathrm{cm}^{3}$ and $\nu=0.145 \mathrm{~cm}^{2} / \mathrm{s}$. The kinematic parameters presented in Sec. 2.1 are set as follows: $f=186 \mathrm{~Hz}, \Delta t_{r}=0.43 T$ during supination and $0.37 T$ during pronation, $\zeta=0.27 T$ during supination and $0.86 T$ during pronation, $\gamma=0, \beta=0$, and $\Phi=75.6^{\circ}$ where $T$ is the flapping period. Other parameters, $\alpha_{d}, \alpha_{u}$ and $\varphi_{0}$, are determined by three restrictions: the wing provides a lift force equivalent to the hoverfly weight, and the drag and the elevation moment are zero.

The computational domain is $31 c \times 31 c \times 33 c$. A nonuniform grid of $289 \times 157 \times 273$ points is used. The minimum spacings in three directions are respectively $\Delta x=0.0385 c$, $\Delta y=0.0333 c$, and $\Delta z=0.0278 c$. Such choice is based on our previous studies (Tian et al., 2013b,a, 2014a). In our simulations, we have conducted validations by varying computational domain, mesh size and time step to make sure that the results presented in this work are independent of these factors. To maintain CFL $<1.0,2000$ time steps in one flapping cycle are used. It takes about 20 hours to calculate a flapping cycle with 8 computational cores on a workstation with Intel Xeon CPU E5-2600 with OpenMPI.

Two main topics will be discussed: (1) effects of alula on equilibrium kinematics, and (2) effects of alula and its phase differences on wing performance. In the first topic, we first determine the equilibrium kinematics for the wings with and without alula. Then we discuss the aerodynamic performance for wings with and without alula at equilibrium kinematics. In the second topic, both the wings with and without alula flap in the same kinematics, but the alula has a phase difference of $45^{\circ}$ ahead and behind the wing. 


\subsection{Effects of alula on equilibrium kinematics}

\subsubsection{Equilibrium parameters, for the wings with and without alula}

In this section, simulations are conducted to find out the equilibrium parameters $\left(\alpha_{d}, \alpha_{u}\right.$ and $\left.\varphi_{0}\right)$ at which the lift is equal to the hoverfly weight, the drag is zero and the pitching moment is zero. The steps to achieve this are: (1) A case at a values of $\alpha_{d}, \alpha_{u}$ and $\varphi_{0}$ is conducted, and the fluid dynamics is (2) The lift, drag and moment are acquired. Based on aerodynamic information, the values of $\alpha_{d}, \alpha_{u}$ and $\varphi_{0}$ are adjusted and a new simulation is performed; and (3) This process is repeated until the lift force equals the hoverfly weight, and the drag and the elevation moment are zero. For each case, six flapping cycles are calculated.

Thirteen testing cases performed for each wing before the equilibrium parameters are determined. For the wing with alula, the equilibrium parameters are $\alpha_{d}=28.0^{\circ}$, $\alpha_{u}=32.6^{\circ}$ and $\varphi_{0}=7.0^{\circ}$. For the wing without alula, the equilibrium parameters are $\alpha_{d}=29.5^{\circ}, \alpha_{u}=33.6^{\circ}$ and $\varphi_{0}=7.0^{\circ}$. Fig. 2 shows the time histories of the stroke angle and pitching angle at equilibrium flight-in a flapping cycle and pitching angle difference with and without alula. It is noted that the wing without alula experiences a larger angle of attack. The increase of the angle of attack is $1.5^{\circ}$ during down stroke and is $1.0^{\circ}$ during upstroke. These observations can be explained as follows. The area of the wing without alula is smaller. It will produce smaller ${ }_{2}$ lift than the wing with alula the same kinematics; as equilibrium parameters of the wing with alula. To generate larger lift, the angle of attack should be larger. On the other hand, the increase of the angle of attack should maintain the drag and moment restrictions. To de this, asymmetric increase of the angle of attack of during upstroke and downstroke is required. The main mechanism of lift increase by changing angle of attack is the Kramer effect (Ellington, 1984d) where changes in angle of attack ereate circulation as the wing moves. Further explanation will be made, when the vortical structures are discussed. 
(a)

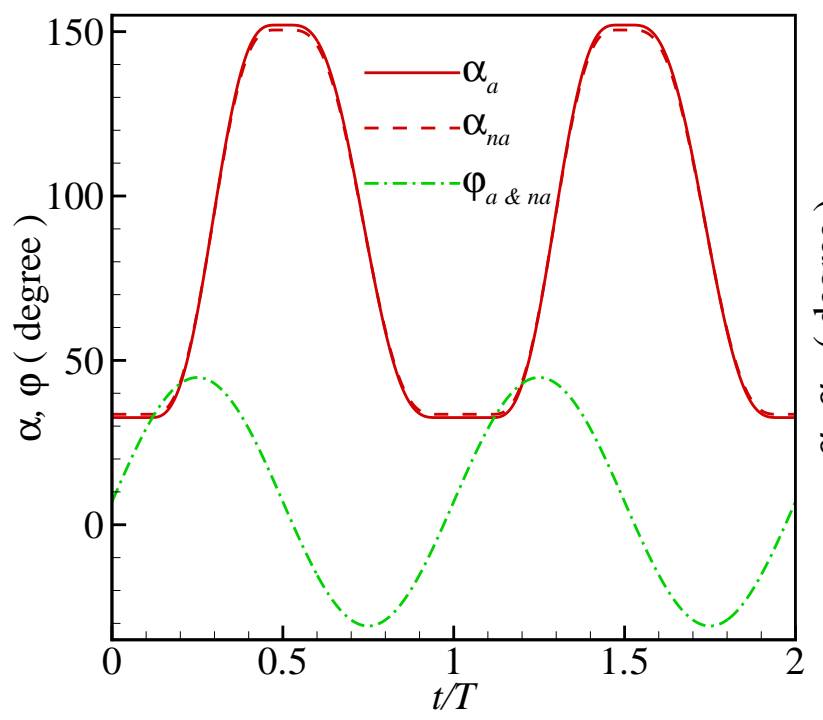

(b)

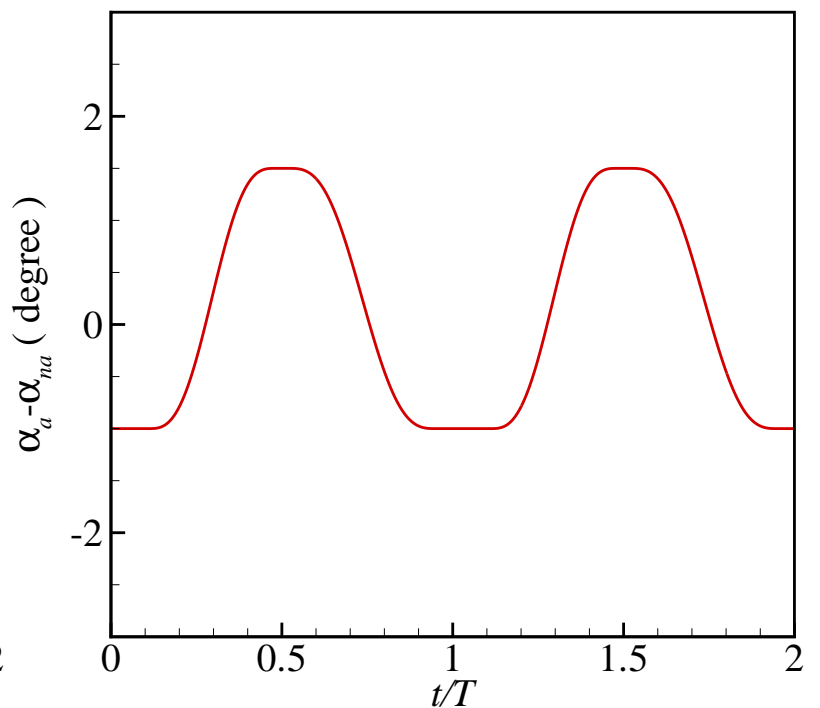

Figure 2: Kinematics at equilibrium-flight: (a) stroke and pitching angles equilibrium flight, and (b) pitching angle difference between wings with and without alula. In the pictures, $\alpha_{a}$ and $\alpha_{n a}$ are respectively the pitching angles of the wings with and without alula.

\subsubsection{Forces at equilibrium parameters}

Here we discuss the forces produced by both wings with and without alula. Fig. 3 shows the time histories of the lift coefficient $C_{L}$, the drag coefficient $C_{D}$, the side force coefficient $C_{S}$ and the elevation moment coefficient $C_{M} \cdot C_{L}, C_{D}$, and $C_{S}$ are respectively the forces in $y, x$ and $z$ directions scaled by $0.5 \rho U^{2} c L$ where $U$ is the mean tip velocity. $C_{M}$ is the moment about the center of mass of the hoverfly in the $z$ direction scaled by $0.5 \rho U^{2} L c^{2}$. Here we use the same area to scale forces due to the fact that the direct contribution of alula (force on alula) is less than $0.2 \%$ compared to that of the whole wing. Five observations are made. First, there are lift peaks translational periods (near $t / T=4$ and 4.5 ) and pitching reversals (near $t / T=4.3$ and 4.8 ). The contribution of the translational motion is much larger compared to the pitching motion. Second, near the end of each stroke (near $t / T=4.25$ and 4.75$)$, there is a lift loss which is caused by the induced flow interacting with the wing. Third, the mean lift coefficient is about 1.372 for the wing with alula and 1.380 for the wing without alula. Based on the experiments in Ref. (Mou et al., 2011), the lift coefficient of each wing is 1.372 so that a pair of wings can support the hoverfly for 
hovering flight. The result here is less than $0.6 \%$ different from the experimental result. Fourth, the mean values of $C_{D}$ and $C_{M}$ are respectively less than 0.03 and 0.05 . Such errors are reasonable when simulations are conducted to seek equilibrium kinematics (Mou et al., 2011). As we only simulate one wing, the mean value of $C_{S}$ is not zero. It is balanced by another wing. Finally, the difference of the force histories between the wings with and without alula is not significant. The difference appears mainly near the translational stroke period. The translational upstroke compensates for the lift drop during downstroke, as shown in Fig. 3(a). Similar compensation mechanism is observed drag and moment. This is the advantage of asymmetric increase of the angle of attack. 
(a)

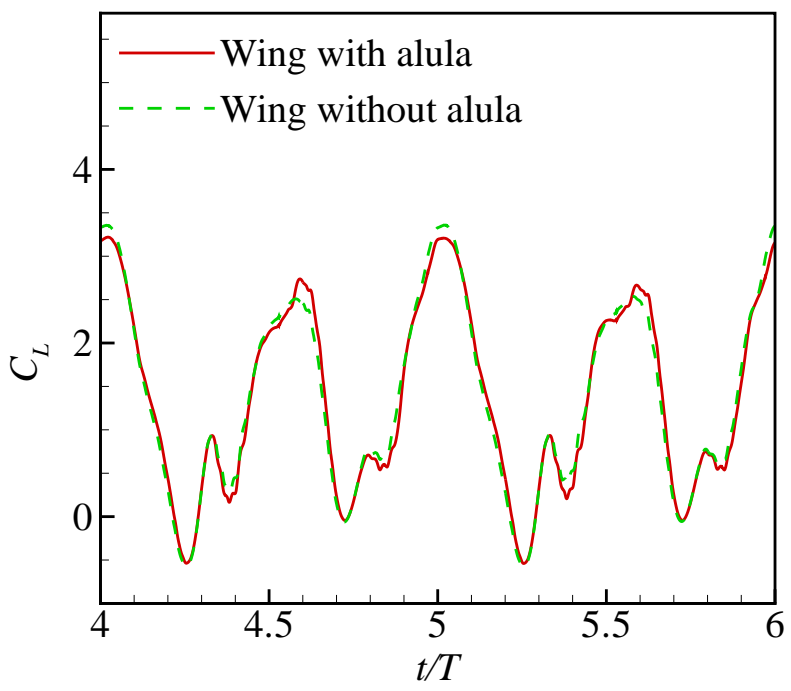

(c)

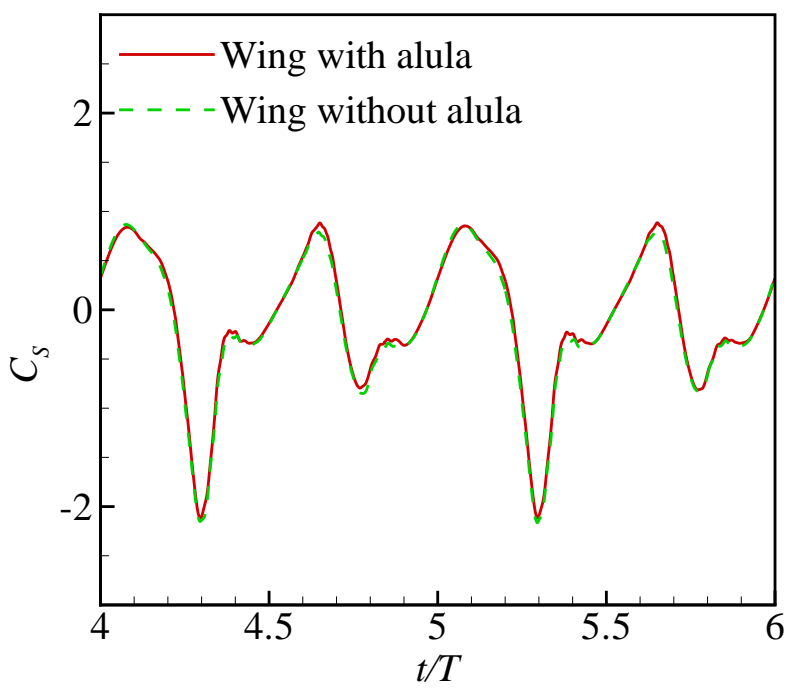

(b)

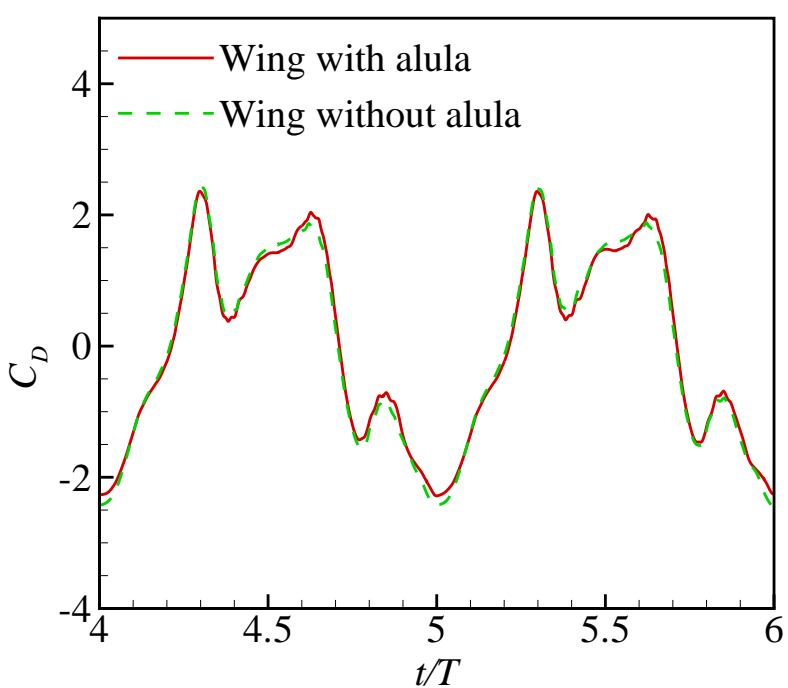

(d)

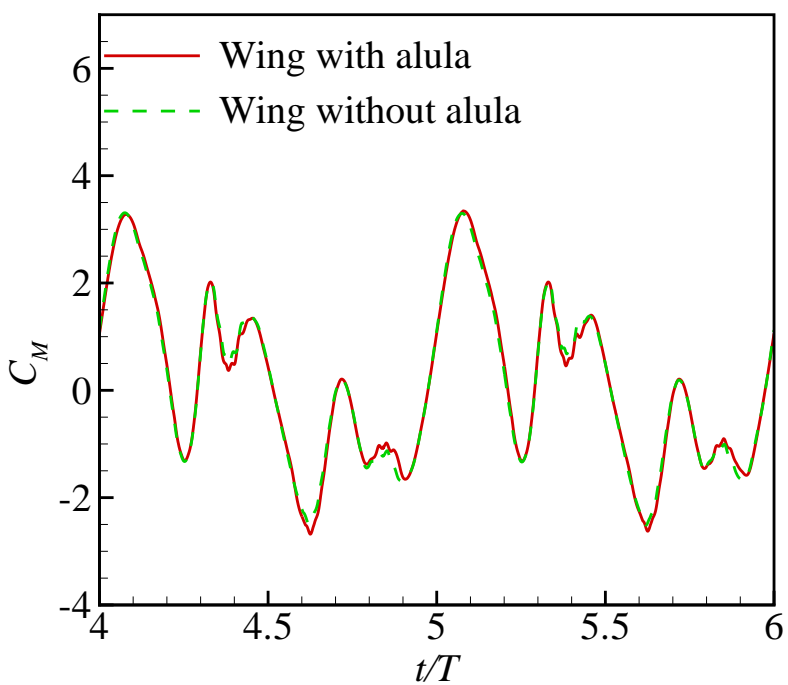

Figure 3: Time histories of the force coefficients on the wings with and without alula: (a) lift coefficient, (b) drag coefficient, (c) side force coefficient, and (d) elevation moment coefficient.

\subsubsection{Aerodynamic power at equilibrium parameters}

Another important quantity to measure the flight performance of an insect is the aerodynamic power, which is shown in Fig. 4. The mean aerodynamic power coefficient is 1.811 for the wing with alula and 1.897 for the wing without alula. The wing without alula needs $5 \%$ more power compared to the wing with alula. The maximum stroke angle is the same for 
both cases. To adjust the kinematics, the wing without alula increases its angle of attack to compensate for the lift drop because of the smaller area. As shown in Fig. 3(a), the lift force of the wing without alula is larger $\operatorname{man}_{\mathbf{A}} 4<t / T<4.08$, and $4.48<t / T<4.58$ compared to that of the wing with alula. The larger forces in these regions cause the dominant increase of the power requirement. There is a power decrease $\operatorname{in}_{\mathbf{2}} 4.58<t / T<4.6$. But such decrease is much smaller compared to the increase during the translational strokes. The power increase is observed for hawkmoth with damaged wings during hovering flight Fernández et al. (2017). Based on this observation, we presume that the natural wing designs are optimal.

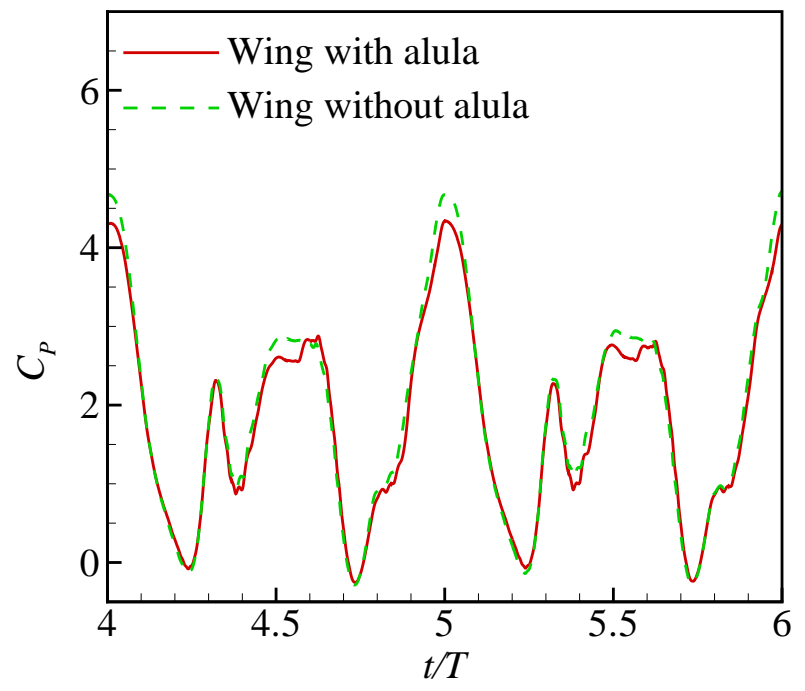

Figure 4: Time histories of aerodynamic power coefficient.

\subsubsection{Vortical structures at equilibrium parameters}

The vortical structures in the near field of a flapping wing is closely related to the forces 丹f the wing (Wu et al., 2006; Deng et al., 2013). Therefore, it is necessary to investigate the vortical structures around the flapping wings. Fig. 5 shows the vortical structures of the wings with and without alula at four instants during a flapping cycle. Similar to the realistic hoverfly case (see Sec. A.2), the distinguished flow features associated with flapping wings are identified for both wings with and without alula. Overall, the vortical structures for both wings are very similar, but three minor differences are observed. The first observation is that the leading edge and root vortices are shifted outward along the rotation axis and 
weaker for the wing without alula, see Fig. 5 at $3 T / 4$. This is the major reason the lift reduction afterwards. The second observation is that the leading edge vortex for the wing with alula is more stable compared to that of the wing without alula. This is demonstrated in Fig. 5 at $1 T / 4$ and $3 T / 4$, where the leading edge vortex near the wing root for the wing with alula is stronger and closer to the leading edge. The last observation is that there is a RVB (root vortical beam) in the wing without alula, as shown in Fig. 5 at $0 T / 4$. The RVB provides a positive contribution to the lift compensating lift loss near $t / T=4.6$. 
(a)
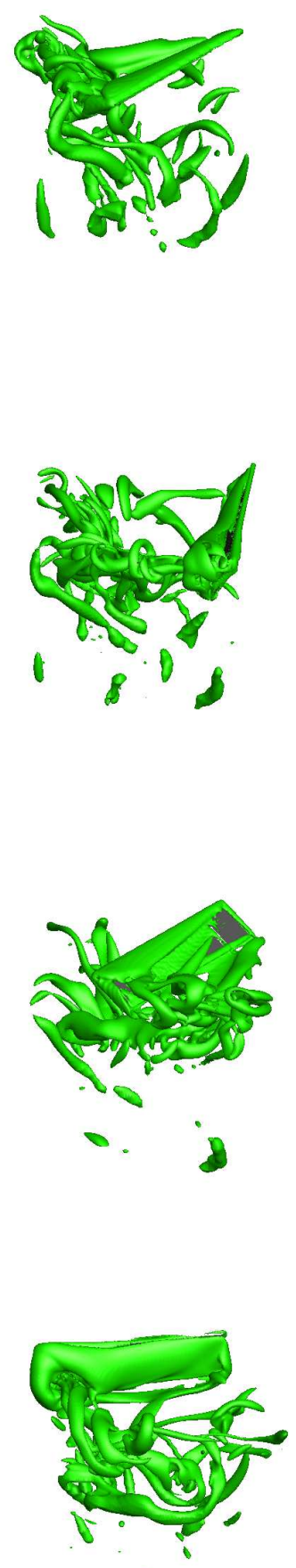

8 (b)
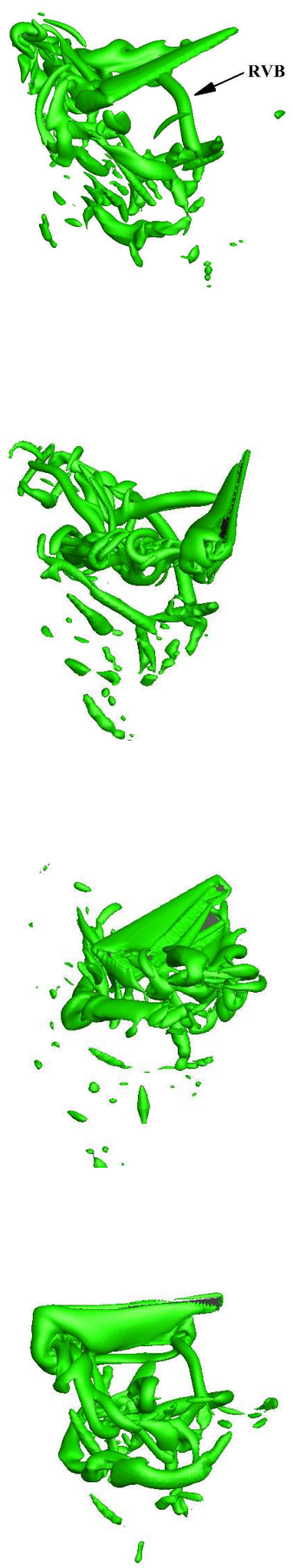

Figure 5: Vortical structures of the wings with alula (a) and without alula (b) at four instants during a flapping cycle. From top to bottom are respectively for $0 T / 4,1 T / 4,2 T / 4$ and $3 T / 4$. 


\subsection{Effects of phase differences on wing performance}

In Sec. 3.1, effects of the alula on the equilibrium kinematics have been discussed by considering two wings with and without alula. For the wing with alula, the alula flaps in phase with the wing. In this section, we will discuss effects of phase differences between the alula and the wing on wing performance. Four cases, wing without alula, wings with the alula flapping in phase, $45^{\circ}$ ahead and $45^{\circ}$ behind the wing, are investigated. All cases are conducted at the same kinematics as that of the wing with alula at equilibrium-flight, i.e. $\alpha_{d}=28.0^{\circ}, \alpha_{u}=32.6^{\circ}$ and $\varphi_{0}=7.0^{\circ}$. By doing this, a continuous approximation is adopted to model the flipped and flat modes (Walker et al., 2012).

\subsubsection{Forces and power}

Figs. 6 and 7 show the time histories of the force coefficients and aerodynamic power coefficient for the wing without alula and the wings with alula flapping in different phases. From these figures, several interesting observations are made. First, the mean lift coefficients for the wings with the alula flapping in phase, $45^{\circ}$ ahead ${ }_{1}$ and $45^{\circ}$ behind the wing and wing without alula are respectively $1.372,1.359,1.334$ and 1.331 . It shows that the wing with alula flapping in phase produces largest lift. Second, at the translational periods (mid downstroke, $t / T=4.0$, and mid upstroke, $t / T=4.5$ ), the wing with alula flapping $45^{\circ}$ ahead produces largest lift, followed by the wing with alula flapping in phase, the wing with alula flapping $45^{\circ}$ behind, and the wing without alula producing smallest lift. This observation can be explained by the contribution of the alula with different phases. The alula flapping $45^{\circ}$ ahead experiences larger angle of attack, followed by the one flapping in phase and the one flapping $45^{\circ}$ behind. Similar to the discussion in Sec. 3.1, the alula with a larger angle of attack will produces larger lift. In addition, the $45^{\circ}$-ahead flapping alula acts as a device to form a downward bow deformation. Similar observation has been made for the force coefficient in the $x$ direction, $C_{D}$. Third, the-wing with alula flapping $45^{\circ}$ behind has profound effect on the force generation at the beginning of the stroke, see Fig.6 at $t / T=4.4$ and $t / T=4.9$. This is caused by the larger rotation angle of the alula during supination and pronation. Finally, the mean power coefficients for the wings with the alula flapping in phase, $45^{\circ}$ ahead and $45^{\circ}$ behind the wing and wing without alula are respectively 1.806 , 
1.801, 1.832 and 1.789. The efficiencies measured by the power coefficients divided by the lift coefficients for the wings with the alula flapping in phase, $45^{\circ}$ ahead and $45^{\circ}$ behind the wing and wing without alula are respectively $0.760,0.755,0.728$ and 0.744 . Therefore, the same kinematics considered here, the efficiency of the wing with alula flapping in phase is lowest and the wing with alula flapping $45^{\circ}$ behind has the highest efficiency. The difference between the best performance and worst performance is about 4\%. The force production could be enhanced by using machine learning algorithms to tune the alula kinematics or optimizing parameters. 
(a)

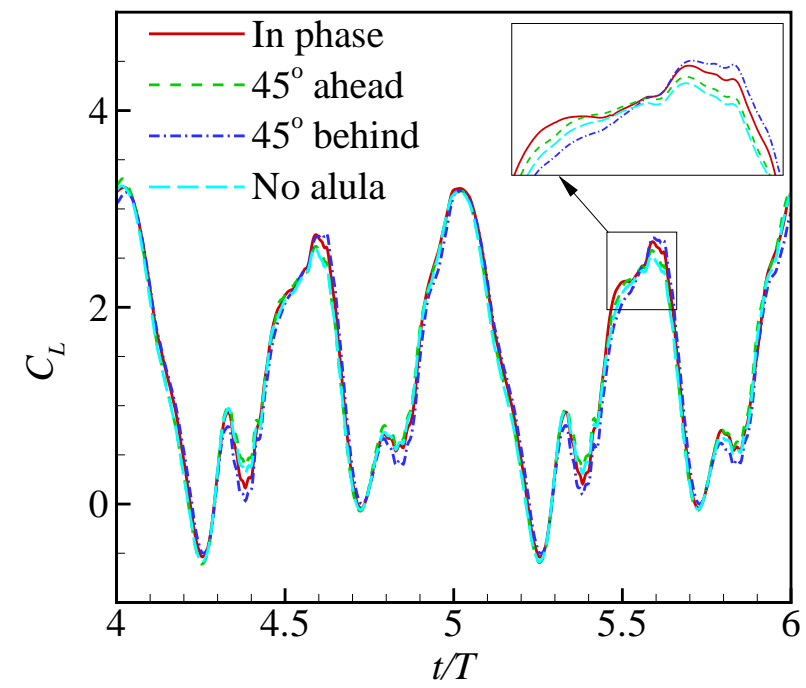

(c)

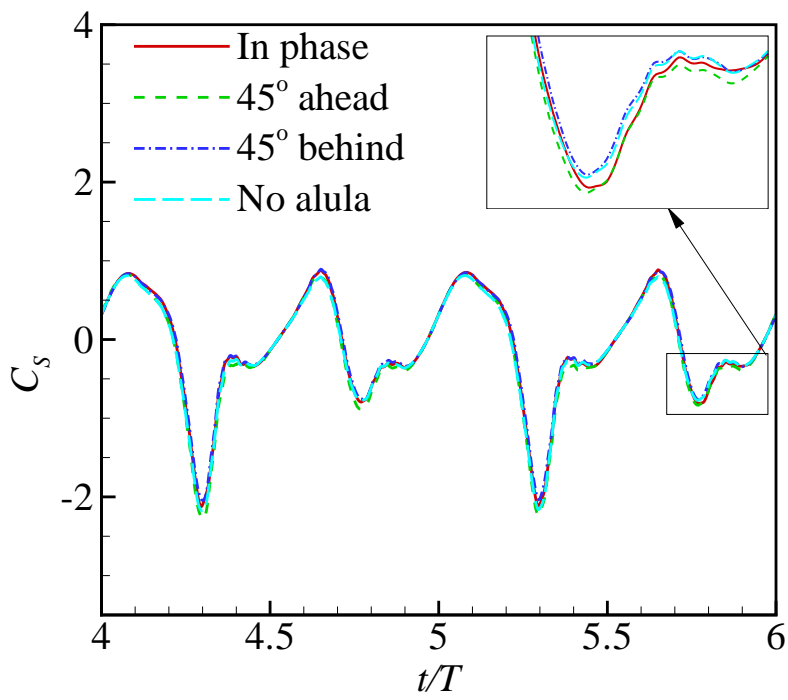

(b)

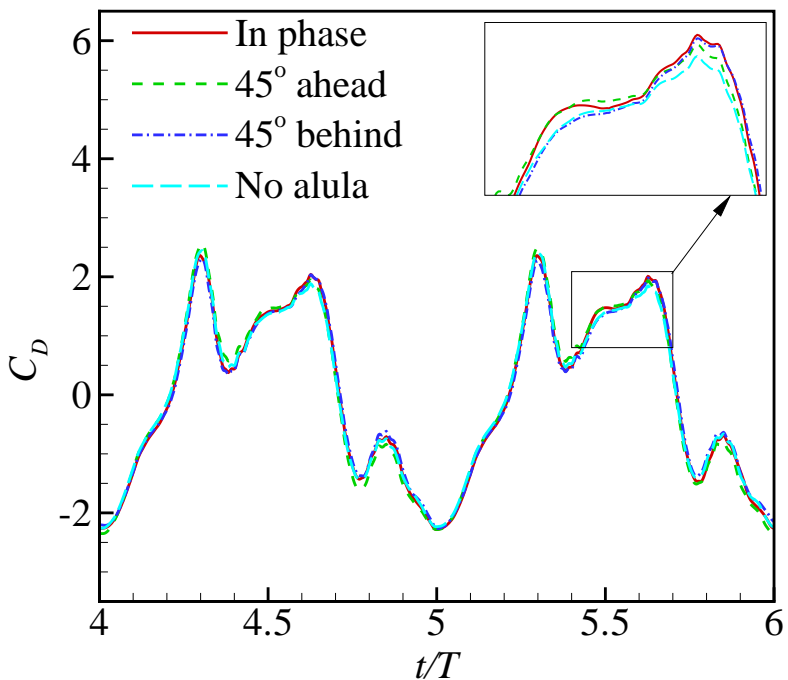

(d)

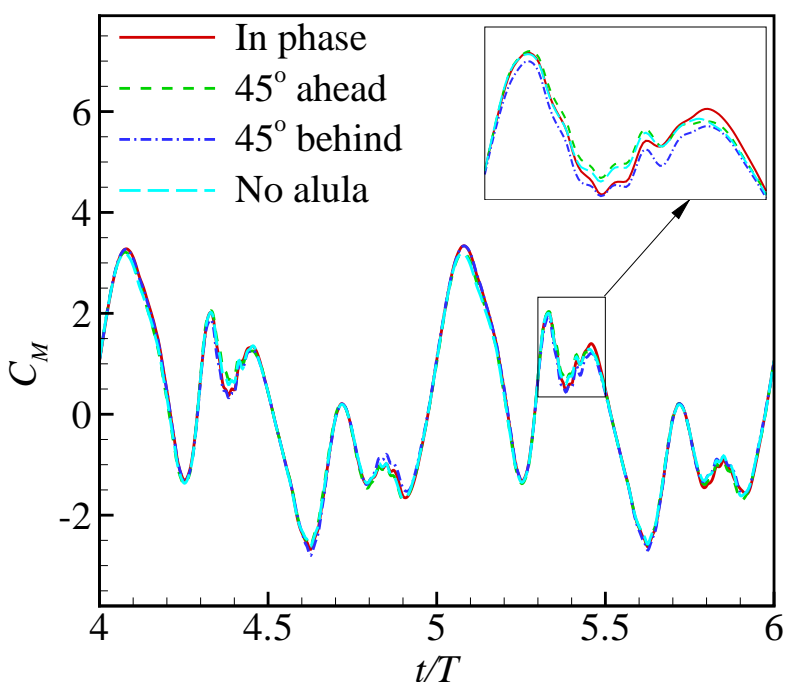

Figure 6: Time histories of the force coefficients for the wing without alula and the wings with alula flapping in different phases: (a) lift coefficient, (b) drag coefficient, (c) side force coefficient, and (d) elevation moment coefficient. 


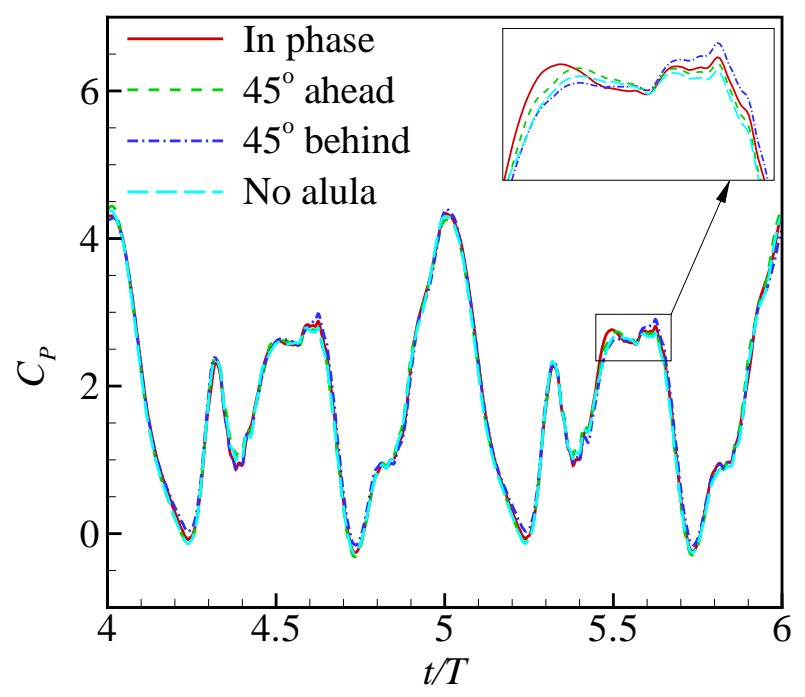

Figure 7: Time histories of aerodynamic power coefficient for the wing without alula and the wings with alula flapping in different phases.

\subsubsection{Vortical structures}

The vortical structures of the wings with alula flapping (a) $45^{\circ}$ ahead, (b) $45^{\circ}$ behind, and (c) the wing without alula are shown in Fig. 8. It is noted that the vortical structures of the wing without alula are almost the same as those shown in Fig. 5(b), which means that the difference between the equilibrium kinematics of both wings in Sec. 3.1 does not significantly affect the flow field. The vortical structures of the wing with alula flapping $45^{\circ}$ ahead are similar to those of the wing without alula. A notable difference is that the leading edge vortex of the wing with alula flapping $45^{\circ}$ ahead is more stable, as shown in Fig. 8(a) and (c) at $t / T=3 / 4$. Such similarity is demonstrated in Fig. 6(a), where the lift coefficients of these two cases are closer compared to other cases as shown by the inset. 
(a)
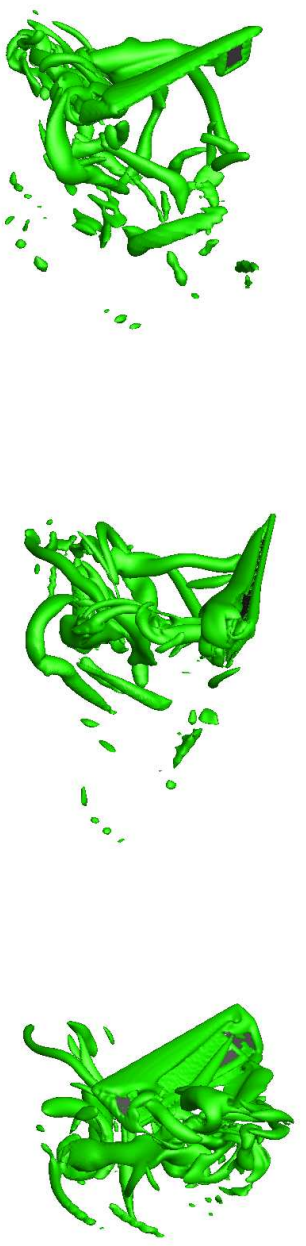

e
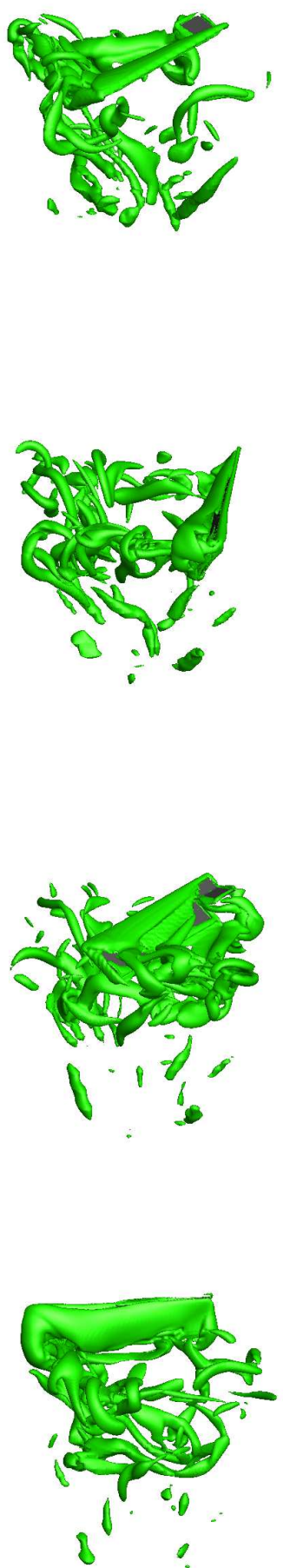

(c)
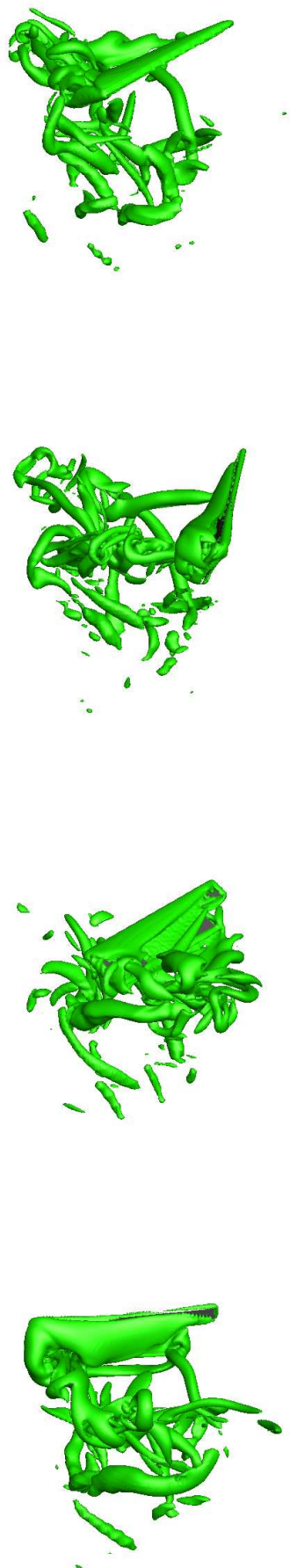

Figure 8: Vortical structures of the wings with alula flapping (a) $45^{\circ}$ ahead, (b) $45^{\circ}$ behind, and (c) the wing without alula. From top to bottom are respectively for $0 T / 4,1 T / 4,2 T / 4$ and $3 T / 4$. 


\section{Concluding remarks}

In this paper, a recently developed flow solver based on the sharp-interface immersed boundary method has been first introduced. The flow field around a realistic hoverfly with prescribed kinematics during hovering flight, has been conducted to validate the solver.

Aerodynamic characteristics of generic wings during hovering flight have been presented. Simulations have been conducted in two groups: One group of simulations have been conducted to determined the equilibrium kinematics of the wings with and without alula; The other group of simulations have been conducted to study the effects of pitching phase differences between the wing and the alula on the performance of the wings. The lift, drag, side force, aerodynamic power, efficiency and vortical structures are discussed in detail. It is found that the wing without alula experiences ${ }_{\perp}$ larger angle of attack at equilibrium flight and requires $5 \%$ more aerodynamic power compared to the wing with alula. The wing with alula flapping in phase produces the largest lift, but its efficiency is lowest. Vortical structure analysis shows that the vortical structures of the wing with alula flapping $45^{\circ}$ ahead are similar to those of the wing without alula and the alula provides stabilizing effect on the leading edge vortex for the wings with alula flapping $45^{\circ}$ ahead and in phase. The results suggest that is a promising method to produce high lift by enhancing and stabilizing leading edge vortex. It is observed that the RVB (root vortical beam) appears in all cases except

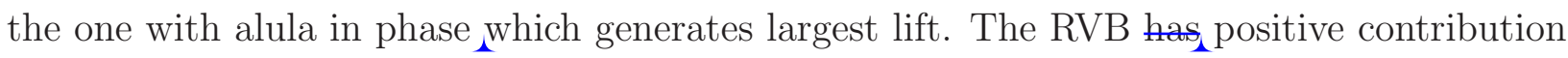
to lift production. This is a reasonable aerodynamic explanation hoverfly rapidly switches alula moving modes. Further effort should be made to clarify the RVB role and its interaction with the hoverfly body.

\section{Acknowledgments}

We thank Dr Richard J. Bomphrey (Department of Zoology, University of Oxford) for his assistance in providing the hoverflyer data and the helpful comments on the paper. This research was supported by the Australian Research Council's Discovery Project Funding Scheme (project number DP130103850). Dr. F.-B. Tian is the recipient of an Australian Research Council Discovery Early Career Researcher Award (project number 
DE160101098). Simulations were undertaken with computational resources on the National Computational Infrastructure National Facility through the National Computational Merit Allocation Scheme supported by the Australian Government.

\section{A. Numerical method and validation}

\section{A.1. Numerical method}

The numerical method used in this work is based on an immersed-boundary method initially developed in Ref. (Mittal et al., 2008) and later improved in Refs. (Luo et al., 2012; Tian et al., 2014a). Only a brief introduction of the method is provided here. The NavierStokes equations are discretized on a nonuniform Cartesian. The momentum equation is integrated in time using the three-step projection method. In the first substep, an advectiondiffusion equation is solved in the absence of the pressure, and an intermediate velocity field is obtained. In this step, both the nonlinear advection terms and the viscous terms are discretized using the Crank-Nicolson scheme to improve the numerical stability. The spatial derivatives are discretized by a second-order central scheme. The discretized system is solved by using the Gauss-Siedel line relaxation method for the linearized system and using successive substitution for nonlinear iterations. In the second substep, a projection function is solved as an approximation of the pressure, and in the third substep, the velocity is updated by applying divergence-free condition.

The special treatment at the fluid-solid interface is the improved second-order sharpinterface immersed boundary method (Luo et al., 2012; Tian et al., 2014a). It retains the sharp-interface representation of the solid body surface and employs local flow reconstruction to facilitate the finite-difference discretization near the boundary. Fig. 9 shows the twodimensional schematics of this method. When the standard second-order central difference scheme is used to discretize the Navier-Stokes equations in the fluid region, incomplete stencils are encountered near the interface. Specifically, on the nodes immediately next to the interface, as the $(i, j)$ point in Fig. 9(a), the finite-difference stencil will involve nodes that are located inside the solid body, as $(i-1, j)$ and $(i, j-1)$. Two methods can be used to calculate the variables of $(i, j)$. In the first method, the variables of $(i, j)$ can be interpolated 
by using the interpolation stencil shown in Fig. 9(a). A body intercept (BI) point can be found by projecting the $(i, j)$ onto the boundary along the surface normal. The variable, $\varphi$, in the local area around $(i, j)$ is approximated by $\varphi=a_{1} x y+a_{2} x+a_{3} y+a_{4}$, where $a_{1}, a_{2}, a_{3}$ and $a_{4}$ can be determined by using the values on BI, together with $(i+1, j),(i, j+1)$, and $(i+1, j+1)$. Then the value on $(i, j)$ can be obtained by $\varphi_{i, j}=\sum_{m=1}^{4} \beta_{m} \varphi_{m}$ where $\varphi_{m}$ is one of the 4 data points. In the second method, we first apply the extrapolation, and then use finite-difference method. As shown in Fig. 9(b), to calculate the value on $(i, j)$, the values on $(i-1, j)$ and $(i, j-1)$ are first extrapolated, and then that on $(i, j)$ is calculated by using the finite-difference method. Take $(i-1, j)$ as an example, the BI point can be determined by the same way as the interpolation. The image point (IP) can be found by taking the symmetrical point about the boundary. The value on IP can be determined by using the shaded stencil, i.e. the values at previous time step on BI, $(i, j),(i, j+1)$, and $(i-1, j+1)$. Then $\varphi_{i-1, j}^{n}=2 \varphi_{B I}^{n}-\varphi_{I P}^{n}$. Therefore, $\varphi_{i, j}^{n+1}$ can be updated by the finite-difference method. In the practice, the numerical oscillations in the moving boundary problems associated with the sudden change of the stencils can be effectively reduced by applying the hybrid scheme of these two methods. Iteration is required in the cases where the points used to interpolate the unknown values are in the solid region or immediately next to the interface. The this method is second order accurate in space. The versatility of it has been demonstrated by its applications in several problems including fish swimming, insect/bird flight and vocal fold vibration in our previous publications (Tian et al., 2012, 2013b,a, 2014a,b; Shahzad et al., 2016). 
(a)

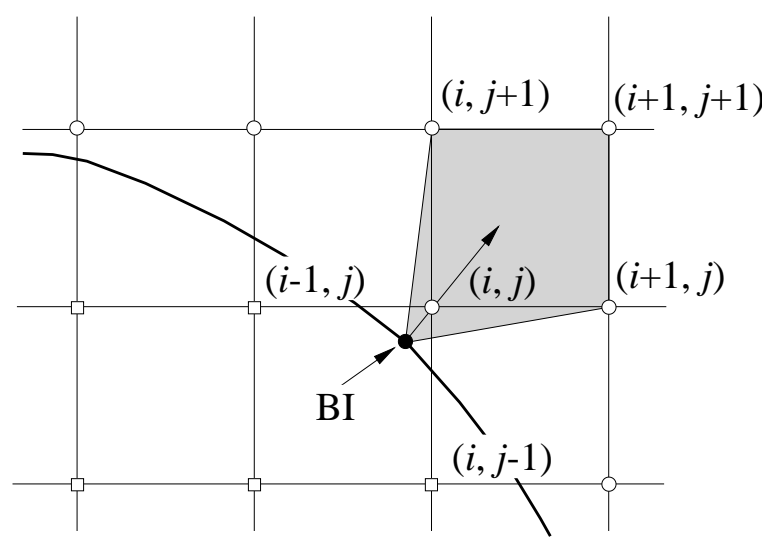

$\square$ Solid point $\circ$ Fluid point (b)

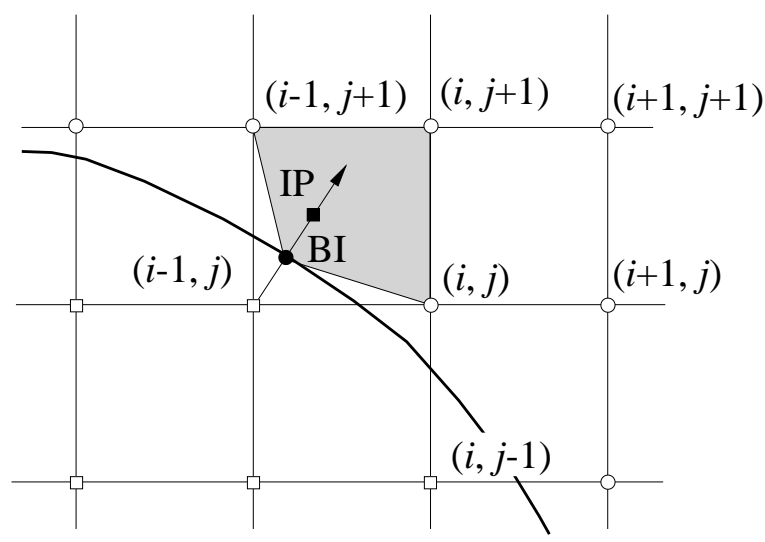

$\square$ Solid point $\circ$ Fluid point

Figure 9: Two-dimensional schematics illustrating the sharp-interface immersed boundary method: (a) interpolation stencil, and (b) extrapolation stencil.

\section{A.2. Validation}

The solver used here has been validated by using simple geometries and kinematics in our previous work (Tian et al., 2012, 2013b,a, 2014a,b; Shahzad et al., 2016). In this work, we further validate our solver by applying it to predict the flow field around a realistic hoverfly with prescribed kinematics. In this application, the hoverfly body motion and the wing deformation were measured by the Animal Flight Group of Department of Zoology at the University of Oxford (Walker et al., 2010, 2012). Specifically, four high-speed digital video cameras were used to reconstruct the motion of approximately 22 points on each wing using photogrammetric techniques. In each flapping cycle, about 26 frames were reconstructed. The cubic spline interpolation is employed to generate the kinematic input for the solver introduced in Sec. A.1. Seven cycles were considered. 


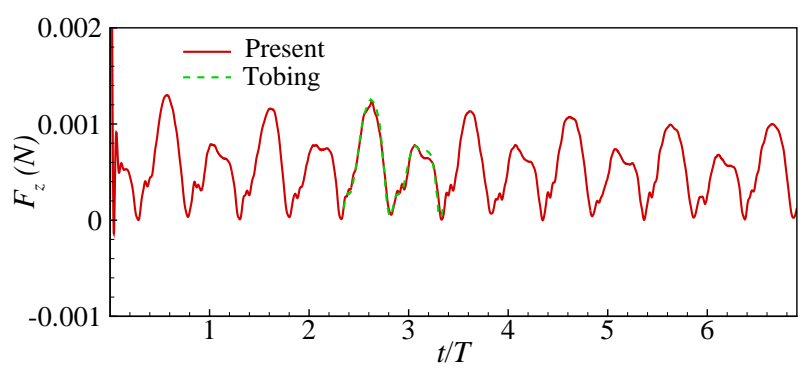

Figure 10: Lift force of the left wing of a realistic hoverfly with prescribed kinematics. The result from Tobing (2014) is shown in the picture for comparison.

The Reynolds number based on the average wing tip velocity $\left(U_{t i p}\right)$ and the wing chord length $(c)$ is about 883 . The computational domain is $26 c \times 30 c \times 27 c$. A nonuniform grid of $293 \times 357 \times 253$ points is used. The minimum spacing is $\Delta x=\Delta y=\Delta z=0.04 c$. In one flapping cycle, 1000 time steps are applied. The lift force of the left wing is shown in Fig. 10. For comparison, the result predicted by Fluent (ANSYS version 14.0) (Tobing, 2014) is shown in the picture. It is found that the present result agrees well with that in the reference. Fig. 11 shows the vortical structures at four instants during the fifth flapping cycle. Here the vortical structure is characterized by the isosurface using the $Q$-criterion defined in Ref. (Hunt et al., 1988). The distinguished flow features associated with flapping wings, such as the leading-edge vortex, the trailing-edge vortex, the tip vortex, and the spanwise flow (Shyy et al., 2010; Deng et al., 2013; Tian et al., 2014a) can be captured by the present flow solver. The validated results here can be used as benchmark case for the future development of methods for complex flows. In future studies, we will investigate the wing-body, wing-wing, and wing-alula interactions and discuss the flight performance in terms of aerodynamic forces and power. In Sec. 3, we will discuss details of the aerodynamic effects of alula by using a generic wing model. 
(a)

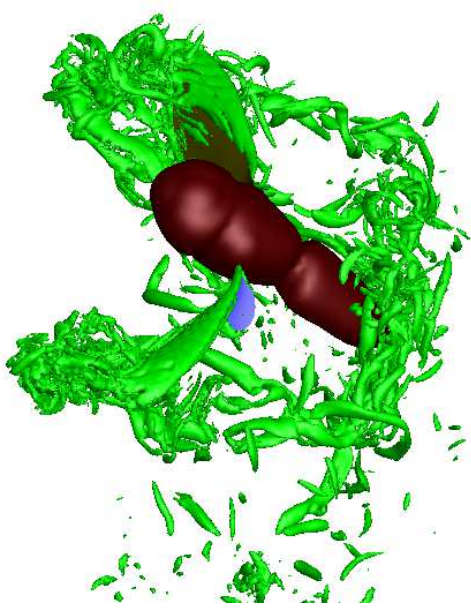

(c)

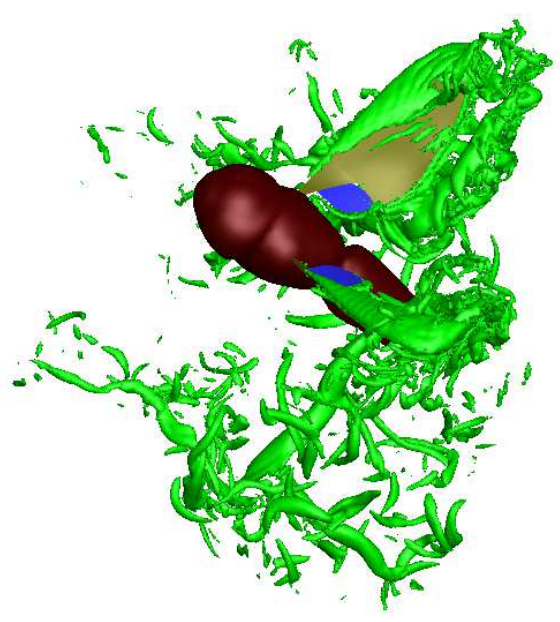

(b)

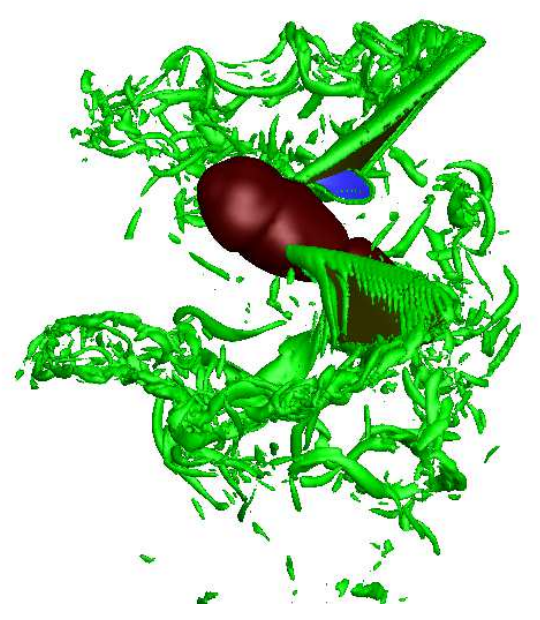

(d)

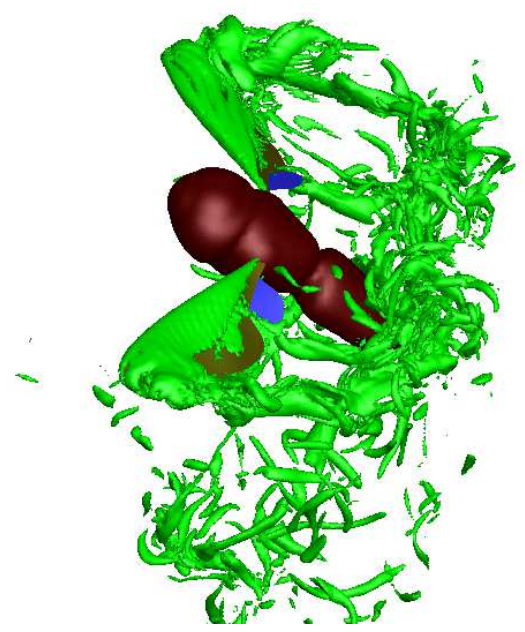

Figure 11: Vortical structures of a realistic hoverfly with prescribed kinematics at four instants during the fifth flapping cycle: (a) mid-upstroke, (b) end of upstroke, (c) mid-downstroke, and (d) end of downstroke.

\section{References}

Alexander, R. M., 1993. Principles of Animal Locomotion. Princeton University Press, New Jersey.

Birch, J. M., Dickinson, M. H., 2002. Spanwise flow and the attachment of the leading-edge 
vortex on insect wings. Nature 412, 729-733.

Deng, H. B., Xu, Y. Q., Chen, D. D., Dai, H., Wu, J., Tian, F. B., 2013. On numerical modeling of animal swimming and flight. Comput. Mech. 52, 1221-1242.

Dickinson, M. H., Lehmann, F. O., Sane, S. P., 1999. Wing rotation and the aerodynamic basis of insect flight. Science 284, 1954-1960.

Dickinson, M. H., Lighton, J. R. B., 1995. Muscle efficiency and elastic storage in the flight motor of Drosophila. Science 268, 87-90.

Dickinson, M. H., Lighton, J. R. B., 2000. How animals move: an integrative view. Science 288, 100-106.

Edwards, R. H., Cheng, H. K., 1981. The separation vortex in the Weis-Fogh circulationgeneration mechanism. J. Fluid Mech. 120, 463-473.

Ellington, C. P., 1984a. The aerodynamics of hovering insect flight. I. The quasi-steady analysis. Phil. Trans. R. Soc. Lond. B 305, 1-15.

Ellington, C. P., 1984b. The aerodynamics of hovering insect flight. II. Morphological parameters. Phil. Trans. R. Soc. Lond. B 305, 17-40.

Ellington, C. P., 1984c. The aerodynamics of hovering insect flight. III. Kinematics. Phil. Trans. R. Soc. Lond. B 305, 41-78.

Ellington, C. P., 1984d. The aerodynamics of hovering insect flight. IV. Aerodynamic mechanisms. Phil. Trans. R. Soc. Lond. B 305, 79-113.

Ellington, C. P., van den Berg, C., Willmott, A., Thomas, A. L. R., 1996. Leading-edge vortices ininsect flight. Nature 384, 626-630.

Fernández, M., Driver, M. E., Hedrick, T. L., 2017. Asymmetry costs: effects of wing damage on hovering flight performance in the hawkmoth manduca sexta. J. Exp. Biol. 220, 36493656. 
Fry, S. N., Sayaman, R., Dickinson, M. H., 2003. The aerodynamics of free-flight maneuvers in Drosophila. Science 200, 495-498.

Hertel, H., 1966. Structure, Form, Movement. Reinhold, New York.

Hunt, J. C. R., Wray, A., Moin, P., 1988. Eddies, stream, and convergence zones in turbulent flows. Center for Turbulence Research Report CTR-S88.

Iosilevskii, G., Joel, D. M., 2013. Aerodynamic trapping effect and its implications for capture of flying insects by carnivorous pitcher plants. Eur. J. Mech. B/Fluids 38, 65-72.

Lighthill, M. J., 1973. On the Weis-Fogh mechanism of lift generation. J. Fluid Mech. 60, $1-17$.

Luo, H., Dai, H., Ferreira de Sousa, P., Yin, B., 2012. On the numerical oscillation of the direct-forcing immersed-boundary method for moving boundaries. Comput. Fluids 56, 6176.

Mittal, R., Dong, H., Bozkurttas, M., Najjar, F. M., Vargas, A., von Loebbecke, A., 2008. A versatile sharp interface immersed boundary method for incompressible flows with complex boundaries. J. Comput. Phys. 227, 4825-4852.

Mou, X., Sun, M., 2012. Dynamic flight stability of a model hoverfly in inclined-stroke-plane hovering. J. Bionic Eng. 9, 294-303.

Mou, X. L., Liu, Y. P., Sun, M., 2011. Wing motion measurement and aerodynamics of hovering true hoverflies. J. Exp. Biol. 214, 2832-2844.

Prempraneerach, P., Hover, F. S., Triantafyllou, M. S., 2003. The effect of chordwise flexibility on the thrust and efficiency of a flapping foil. Proc. 13th Int. Symp. on Unmanned Untethered Submersible Technolog.

Sane, S. P., 2003. The aerodynamics of insect flight. J. Exp. Biol. 206, 4191-4208.

Shahzad, A., Tian, F. B., Young, J., Lai, J. C. S., 2016. Effects of wing shape, aspect ratio and deviation angle on aerodynamic performance of flapping wings in hover. Phys. Fluids $28,111901$. 
Shyy, W., Aono, H., Chimakurthi, S. K., Trizila, P., Kang, C. K., Cesnik, C. E. S., Liu, H., 2010. Recent progress in flapping wing aerodynamics and aeroelasticity. Progr. Aerospace Sci. 46, 284-327.

Shyy, W., Lian, Y., Tang, J., Viieru, D., Liu, H., 2008. Aerodynamics of Low Reynolds Number Flyers. Cambridge University Press, New York.

Tian, F. B., Chang, S., Luo, H., Rousseau, B., 2013a. A 3D numerical simulation of wave propagation on the vocal fold surface. In: Proceedings of the 10th International Conference on Advances in Quantitative Laryngology, Voice and Speech Research. Cincinnati, Ohio, p. 94921483.

Tian, F. B., Dai, H., Luo, H., Doyle, J. F., Rousseau, B., 2014a. Fluid-structure interaction involving large deformations: 3D simulations and applications to biological systems. J. Comput. Phys. 258, 451-469.

Tian, F. B., Lu, X. Y., Luo, H., 2012. Onset of instability of a flag in uniform flow. Theor. Appl. Mech. Lett. 2, 022005.

Tian, F. B., Luo, H., Song, J., Lu, X. Y., 2013b. Force production and asymmetric deformation of a flexible flapping wing in forward flight. J. Fluids Struct. 36, 149-161.

Tian, F. B., Young, J., Lai, J. C. S., 2014b. Improving power-extraction efficiency of a flapping plate: From passive deformation to active control. J. Fluids Struct. 51, 384-392.

Tobing, S., 2014. Numerical analysis of effects of aeroelasticity on flapping-wing propulsion: Case study of bumblebees and hoverflies. Ph.D. thesis, University of New South Wales.

Triantafyllou, M. S., Techet, A. H., Hover, F. S., 2004. Review of experimental work in biomimetic foils. IEEE J. Ocean. Eng. 29, 585-594.

Vogel, S., 1994. Life in Moving Fluids, 2nd Edition. Princeton University Press, New Jersey.

Walker, S. M., Thomas, A. L., Taylor, G. K., 2012. Operation of the alula as an indicator of gear change in hoverflies. J. R. Soc. Interface 9, 1194-1207. 
Walker, S. M., Thomas, A. L. R., Taylor, G. K., 2010. Deformable wing kinematics in free-flying hoverflies. J. R. Soc. Interface 7, 131-142.

Weis-Fogh, T., 1973. Quick estimates of flight fitness in hovering animals, including novel mechanisms for lift production. J. Exp. Biol. 59, 169-230.

Wootton, R. J., 1999. Invertebrate paraxial locomotory appendages: Design, deformation and control. J. Exp. Biol. 202, 3333-3345.

Wootton, R. J., Herbert, R. C., Young, P. G., Evans, K. E., 2003. Approaches to the structural modelling of insect wings. Phil. Trans. R. Soc. Lond. B 358, 1577-1587.

Wu, J. Z., Ma, H. Y., Zhou, M. D., 2006. Vorticity and Vortex Dynamics. Springer-Verlag, Berlin.

Wu, T. Y., 2011. Fish swimming and bird/insect flight. Annu. Rev. Fluid Mech. 43, 25-58.

Xiong, C., Liu, H., Huang, Y., Xiong, Y. (Eds.), 2008. Intelligent Robotics and Applications: First International Conference. Springer-Verlag, New York. 